\title{
The Quantitative Analysis of Alpha-1- Antitrypsin Glycosylation Isoforms in HCC Patients Using LC-HCD-PRM-MS
}

Haidi Yin ${ }^{1,2 \ddagger}$, Jianhui Zhu ${ }^{3 \ddagger}$, Mengmeng Wang ${ }^{3 \dagger}$, Zhong-Ping Yao ${ }^{1,2}$ and David M. Lubman ${ }^{3 *}$

1 The Hong Kong Polytechnic University Shenzhen Research Institute, Shenzhen, PR China

2 Department of Applied Biology and Chemical Technology, The Hong Kong Polytechnic University, Hung Hom, Kowloon, Hong Kong

3 Department of Surgery, University of Michigan Medical Center, Ann Arbor, MI 48109

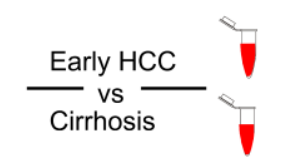

serum

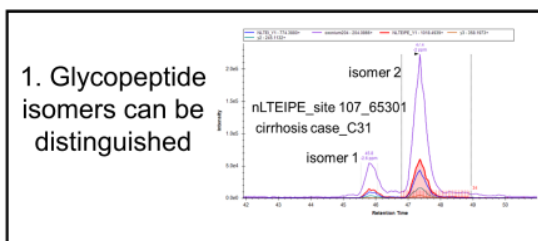

2. Charge states $2+/ 3+$ of one glycopeptide discrimates $\mathrm{HCC}$ from cirrhosis
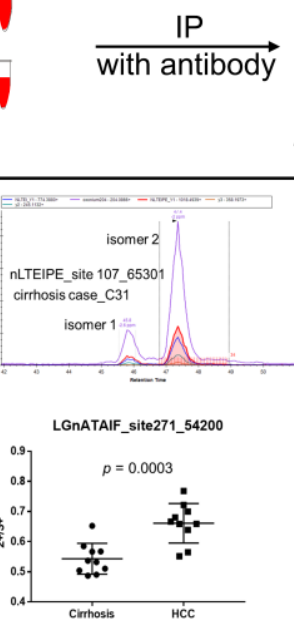

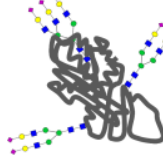

A1AT glycoprotein

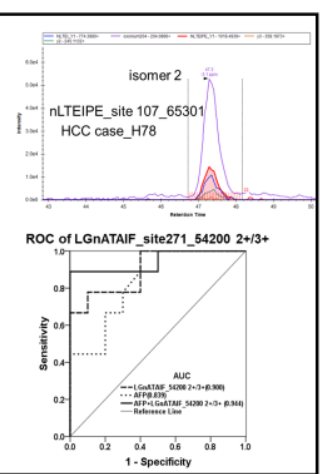

\section{$\underset{\text { Glu-C/chymotrypsin }}{\longrightarrow}$ digestion}

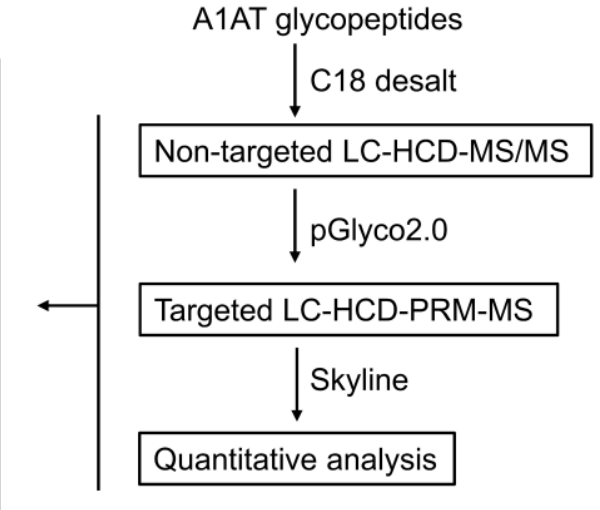

Supporting Information 
Supporting Table S1. Glu-C/chymotrypsin was used to digest A1AT to produce shorter glycopeptides compared with trypsin.

\begin{tabular}{lll}
\hline Glycosylation Site & Trypsin digestion & Glu-C/chymotrypsin digestion \\
\hline Glycopeptide with Asn70 & QLAHQSnSTNIFFSPVSIATAFAMLSLGTK & AHQSnSTNIF \\
Glycopeptide with Asn107 & ADTHDEILEGLNFnLTEIPEAQIHEGFQELLR & nLTEIPE \\
Glycopeptide with Asn271 & YLGnATAIFFLPDEGK & LGnATAIF \\
\hline
\end{tabular}


Supporting Table S2. Reproducibility of the PRM quantification using various transition ions. Reproducibility is expressed as CV\% of the inter-day $(n=3)$ and intra-day $(n=10)$ runs of the same sample. nLTEIPE_65301/nLTEIPE_65300 was used as an example. Y1 ion showed low CV value.

\begin{tabular}{|c|c|c|c|}
\hline $\begin{array}{c}\text { nLTEIPE_65301/ } \\
\text { nLTEIPE_65300 }\end{array}$ & Transition ion & $\begin{array}{l}\text { inter-day } \\
\text { reproducibility } \\
\% \mathrm{CV}(n=10)\end{array}$ & $\begin{array}{l}\text { intra-day } \\
\text { reproducibility } \\
\% \mathrm{CV}(n=3)\end{array}$ \\
\hline \multirow{5}{*}{ oxonium ions } & HexNAc138 & $12.68 \%$ & $1.25 \%$ \\
\hline & HexNAc204 & $9.84 \%$ & $0.66 \%$ \\
\hline & nLTEIPE_01000 (Y1) & $4.13 \%$ & $1.67 \%$ \\
\hline & nLTEIPE_02000 (Y2) & $4.68 \%$ & $3.26 \%$ \\
\hline & nLTEIPE_12000 (Y3) & $5.00 \%$ & $1.20 \%$ \\
\hline \multirow[t]{4}{*}{$Y$ ions } & nLTEIPE_22000 (Y4) & $5.06 \%$ & $3.01 \%$ \\
\hline & nLTEIPE_32000 (Y5) & $3.83 \%$ & $0.92 \%$ \\
\hline & nLTEIPE_33000 (Y6) & $3.76 \%$ & $1.70 \%$ \\
\hline & nLTEIPE_Y1-Y6 & $3.97 \%$ & $1.75 \%$ \\
\hline
\end{tabular}




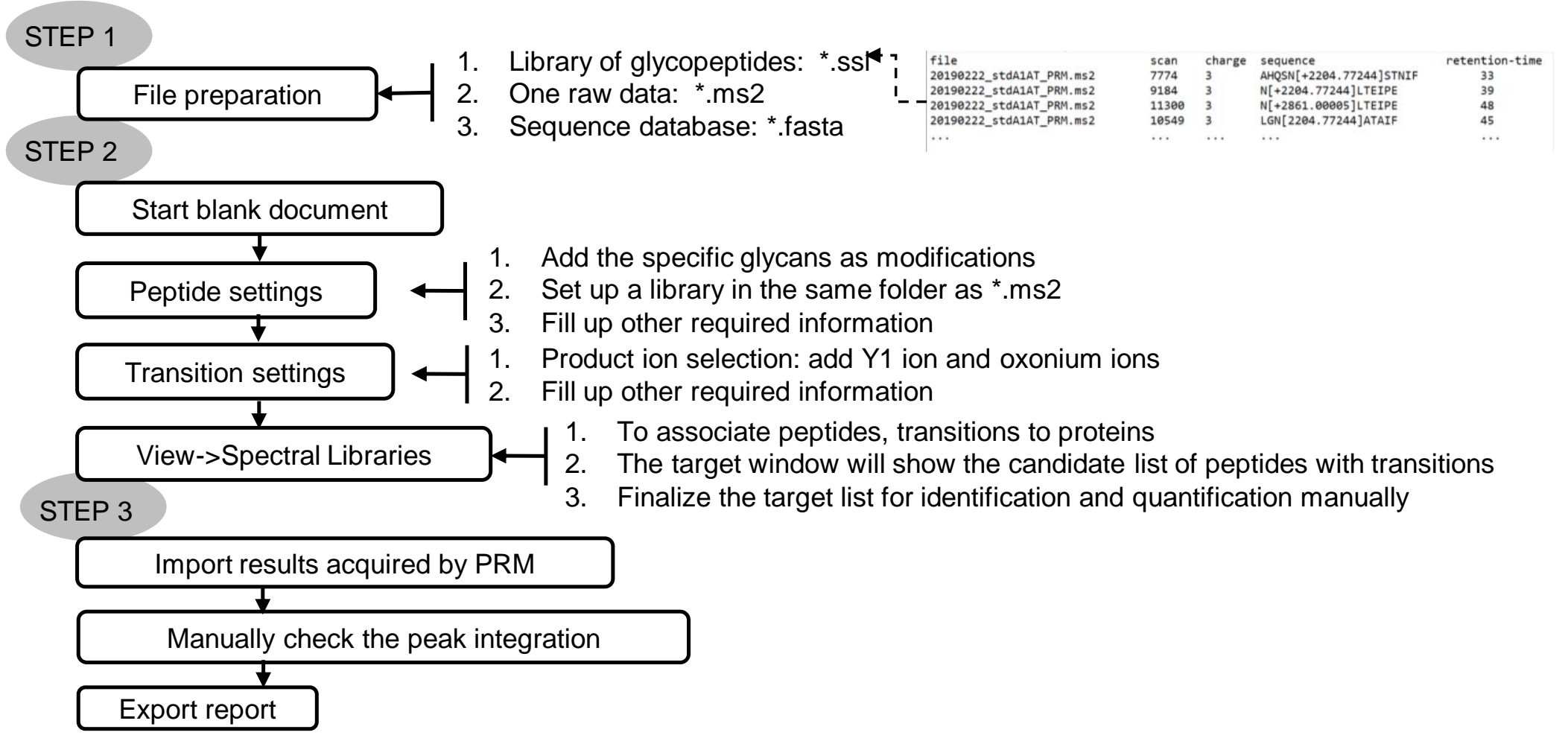

Supporting Figure S1. The Skyline analysis flow chart for the quantification of glycopeptides. 


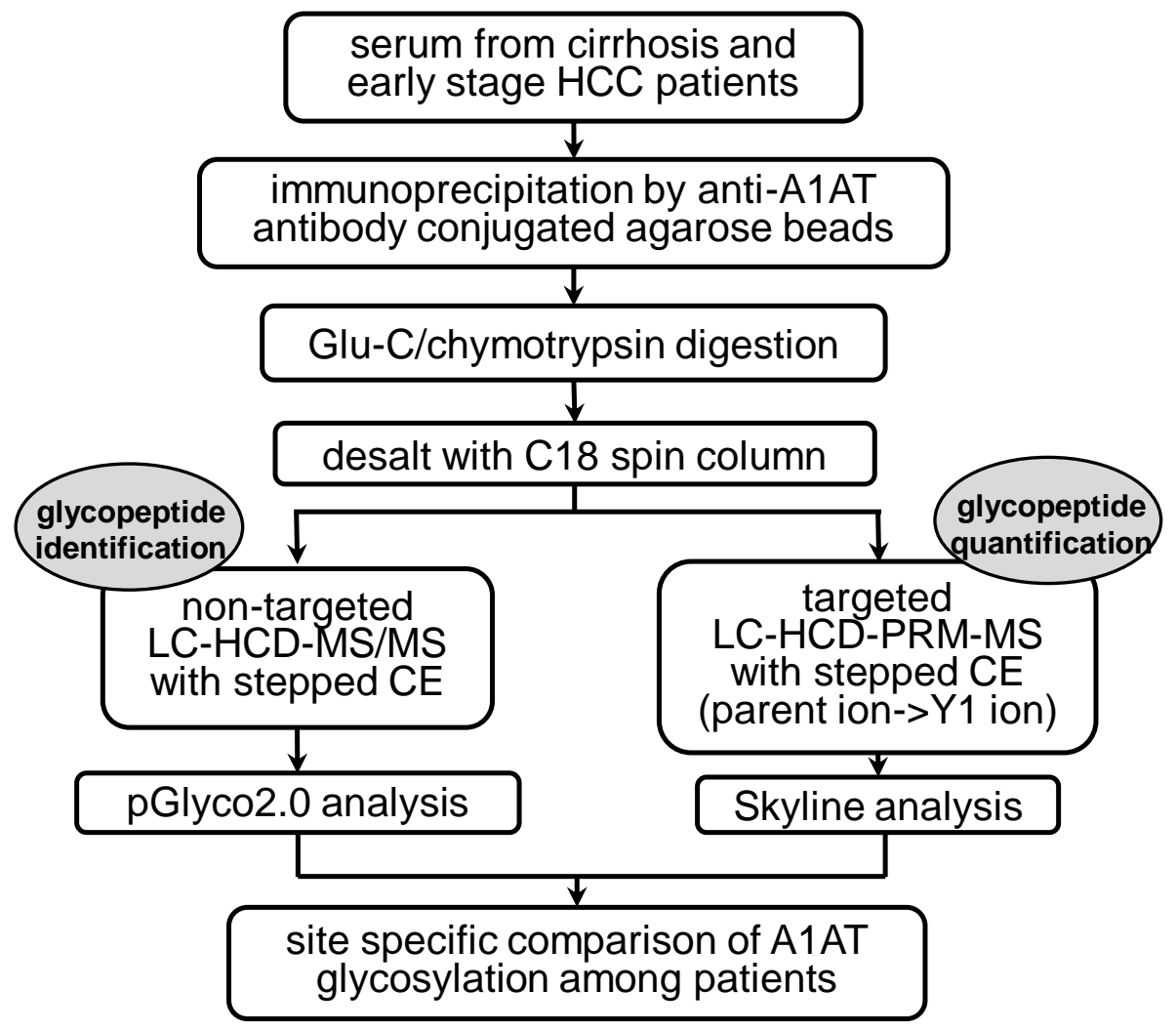

Supporting Figure S2. The experimental workflow to identify glycopeptides and to quantify them by LC-HCD-PRM-MS. 


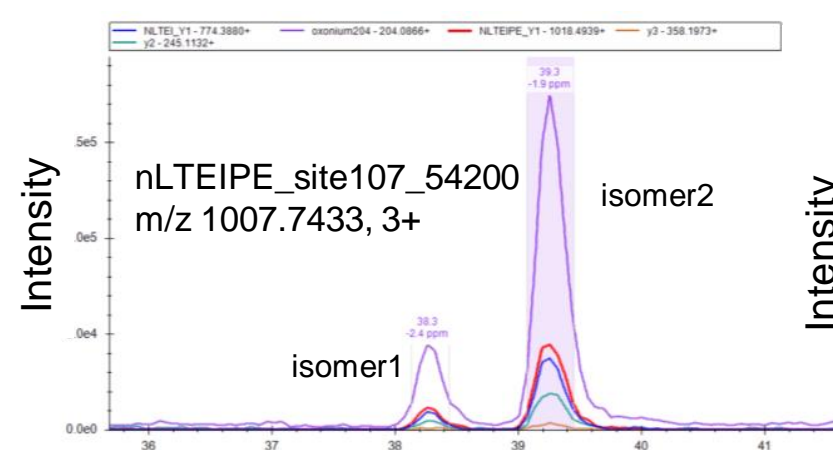

Retention Time

(A)

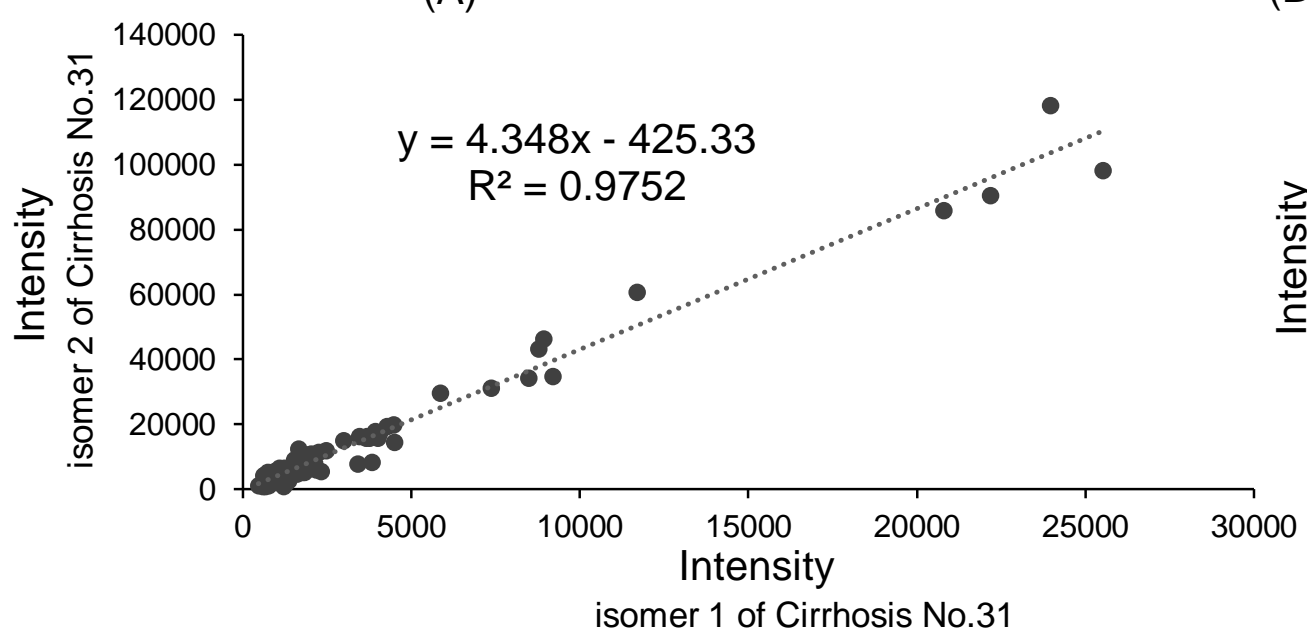

(D)

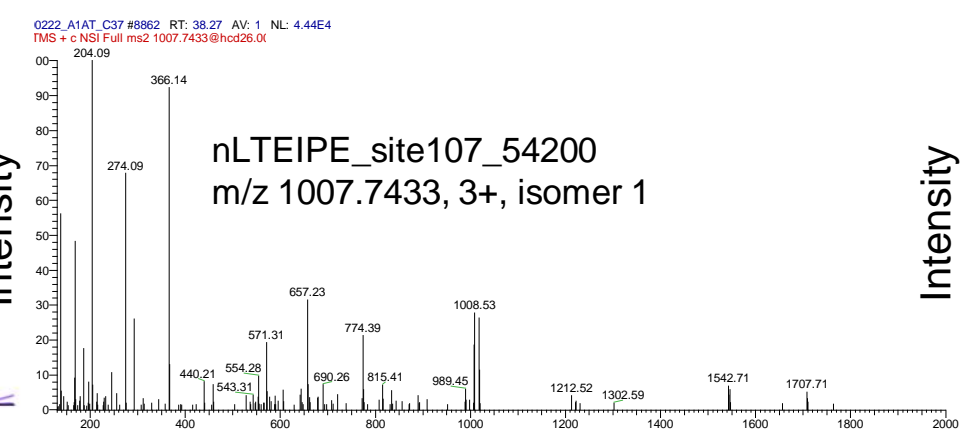

$\mathrm{m} / \mathrm{z}$

(B)

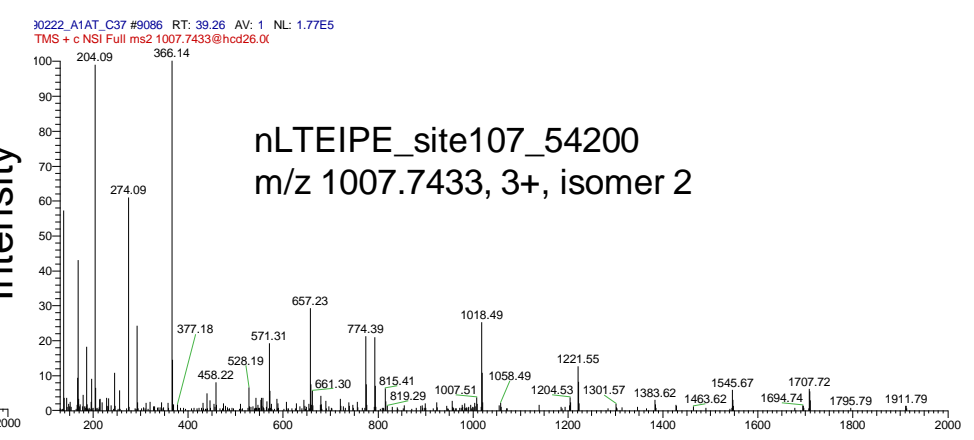

$\mathrm{m} / \mathrm{z}$

(C)

Supporting Figure S3. MS/MS spectra of the two isomers of nLTEIPE_54200 with Asn107. (A) Chromatogram of the two isomers; (B) spectrum of isomer 1; (C) spectrum of isomer 2; (D) The alignment of MS/MS fragments of isomer 1 and isomer2 within the same patient sample; (E) The alignment of MS/MS fragments of isomer 2 from one patient sample and those from another patient sample. (The most abundant top three oxonium ions 366.1383, 204.0861 and 274.0912 have much higher intensity than other fragments and do not show differences so they are excluded from the plot). 


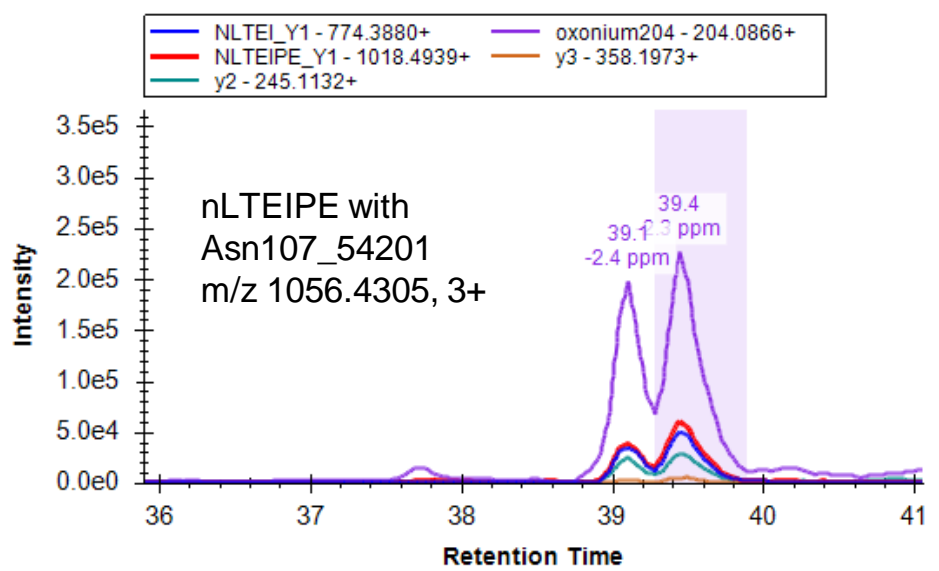

(A)

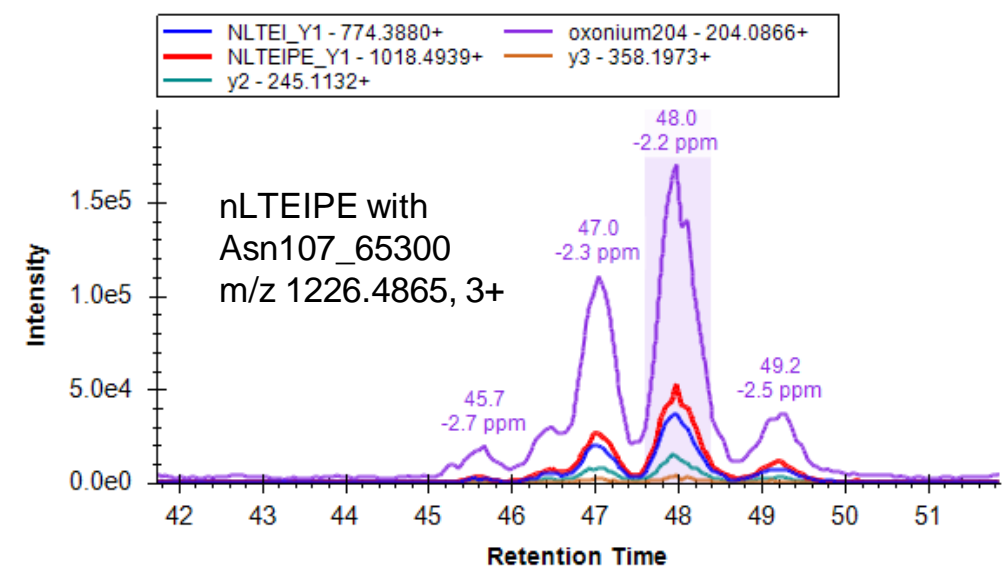

(B)

Supporting Figure S4. Other isomers of nLTEIPE glycopeptide. (A) The two isomers of nLTEIPE_54201 with Asn 107; (B) the four isomers of nLTEIPE_65300 with Asn107. Isomers in both cases were not well separated. 
20190222_A1AT_C31 \#10571 RT: 45.86 AV: 1 NL: 6.13E4

F: FTMS + c NSI Full ms2 1275.1742@hcd26

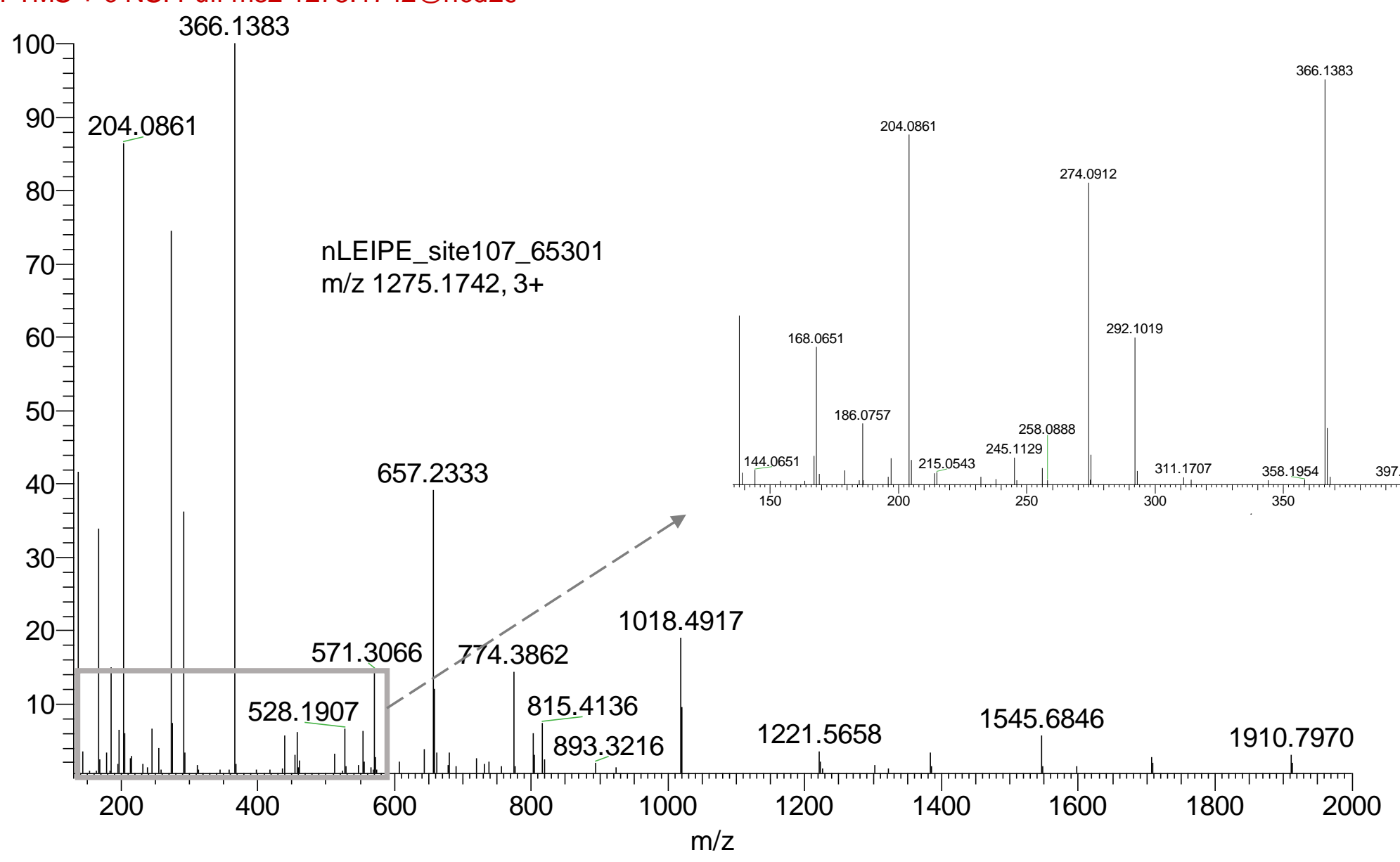

Supporting Figure S5. The MS/MS spectrum of nLEIPE_65301 with site Asn107. It contains oxonium ion 512.1959 , indicating that it is antennary fucosylated. 


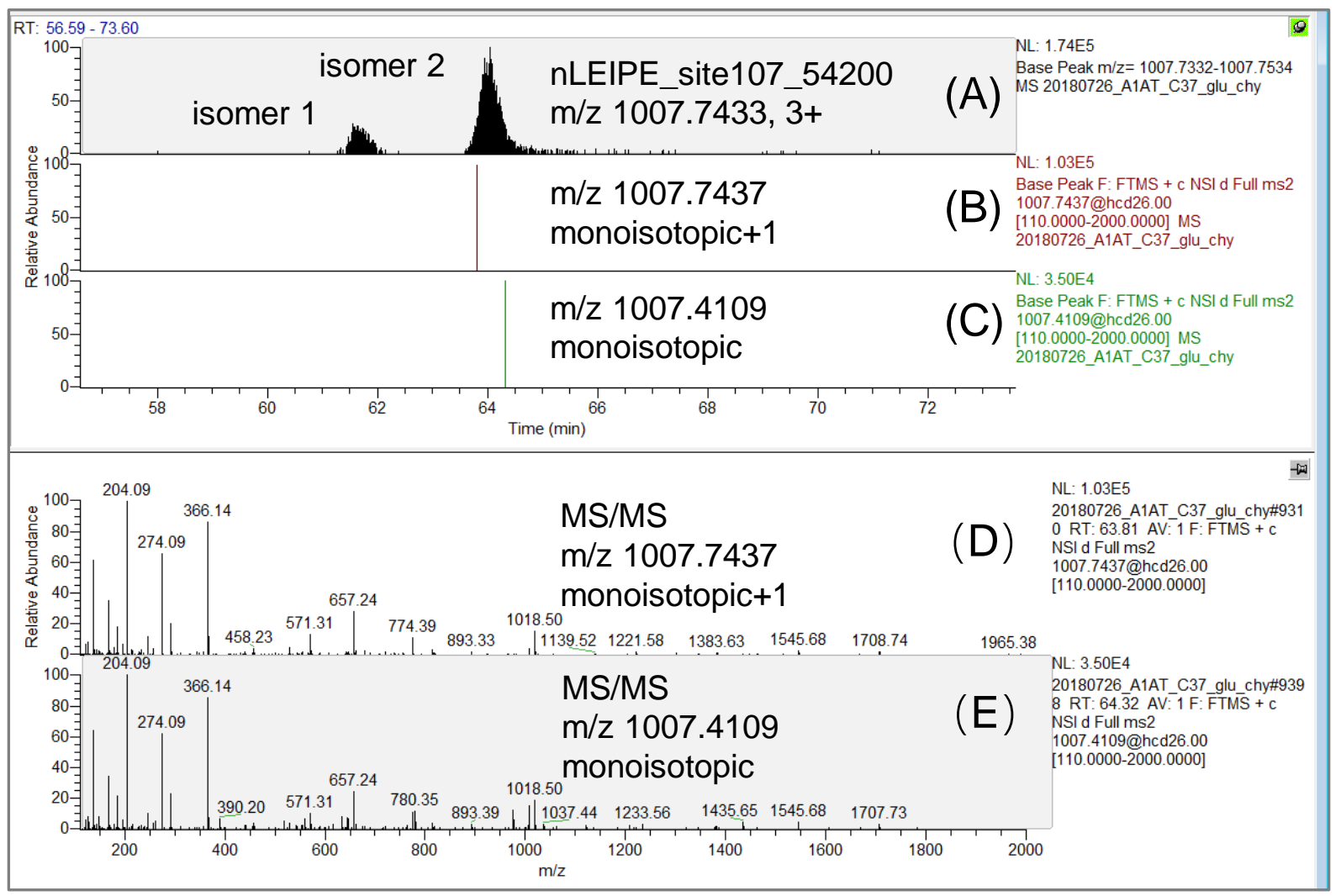

Supporting Figure S6. The importance to quantitate glycopeptides with the PRM strategy. (A) Two isomers of nLEIPE_54200 with Asn107. (B, C) Without the PRM strategy, two MS/MS scans at the elution time of isomer 2 were identified and the quantification was limited to isomer 2. (D, E) The MS/MS spectra of the two scans from isomer 2 were successfully identified; no scan from isomer 1 was identified due to its low intensity. 


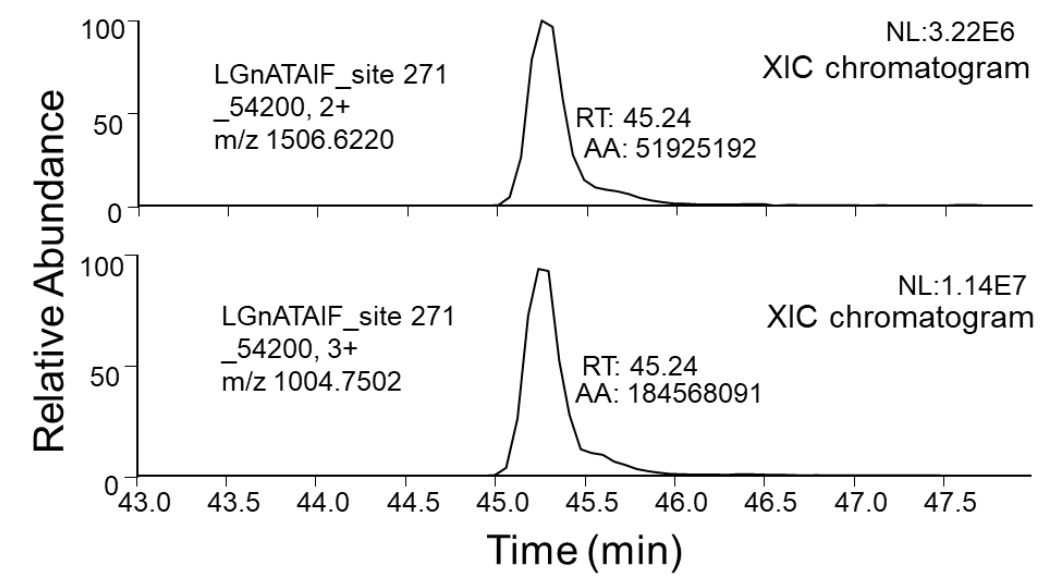

(A)

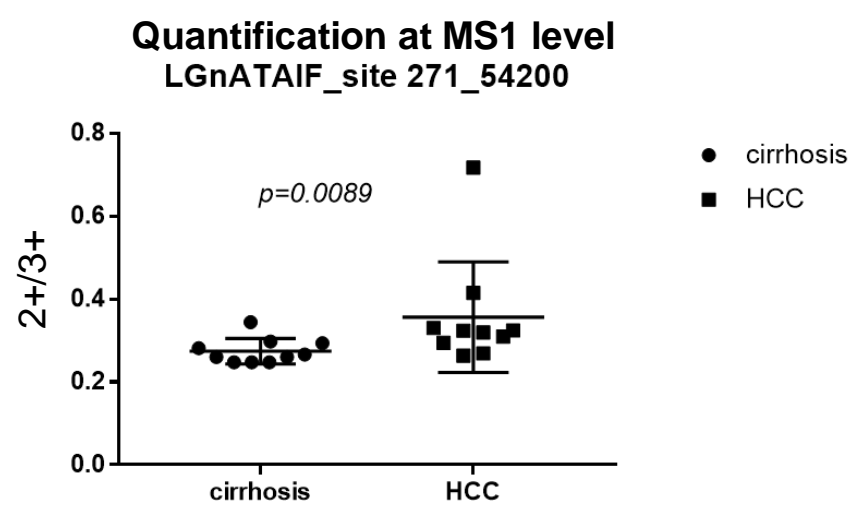

(B)

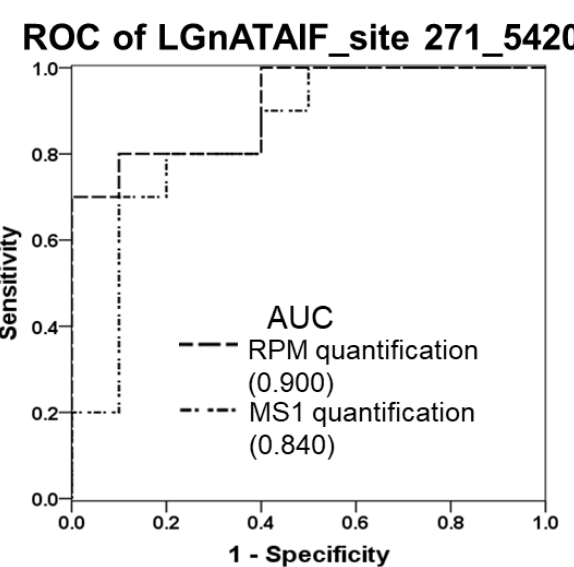

(C)

Supporting Figure S7. The MS1 level analysis of LGnATAIF_54200 with site Asn271 in charge 2+ and 3+ forms. (A) The chromatograms of two different charge states are similar; (B) the boxplot of $2+/ 3+\%$ from MS1 level quantification in cirrhosis and HCC; (C) the ROC analysis of MS1 level quantification 


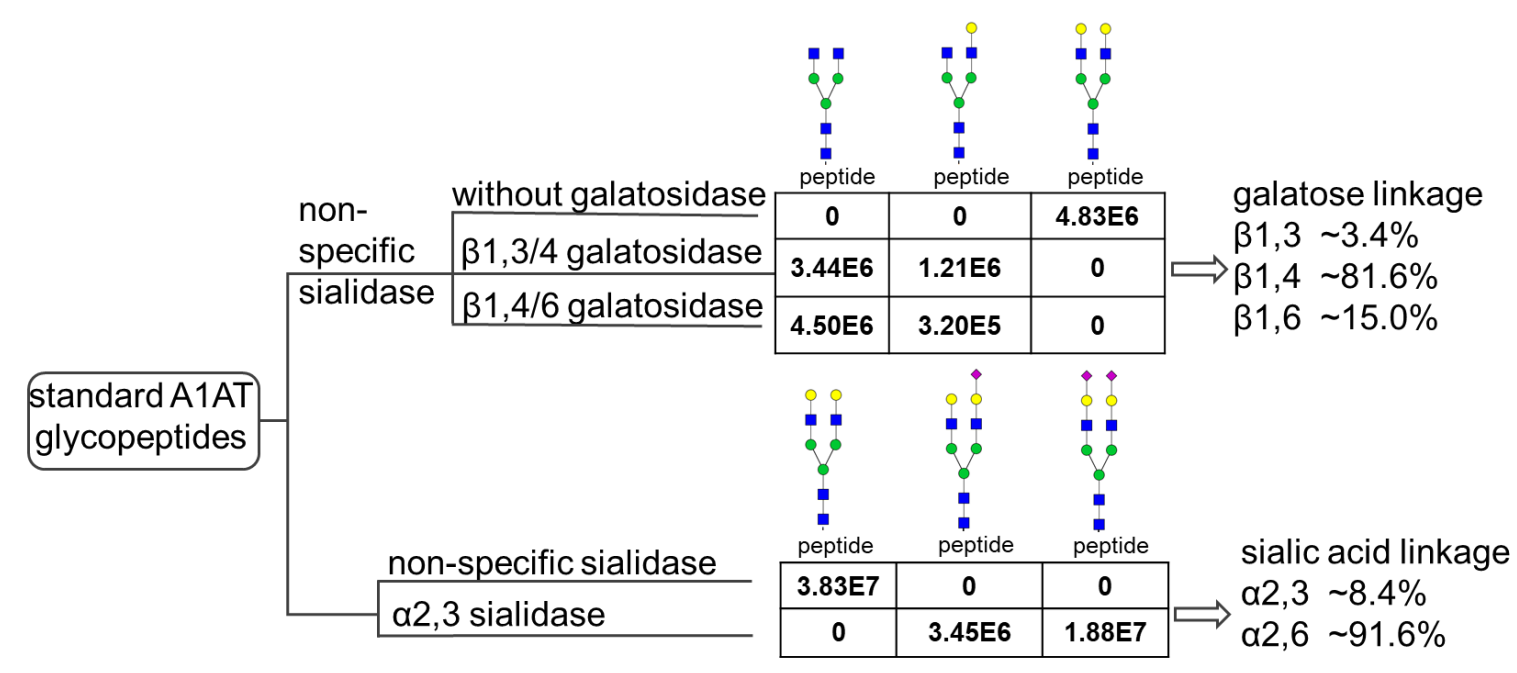

Supporting Figure S8. Exoglycosidase digestion on commercial A1AT standard from human serum for galactose and sialic acid linkage differentiation. A1 AT was first digested with trypsin to glycopeptides, then digested with non-specific/non-specific sialidase and/or specific galactosidases distinguish the relative abundance of sialic acid linkages and galactose linkages. 
(A)
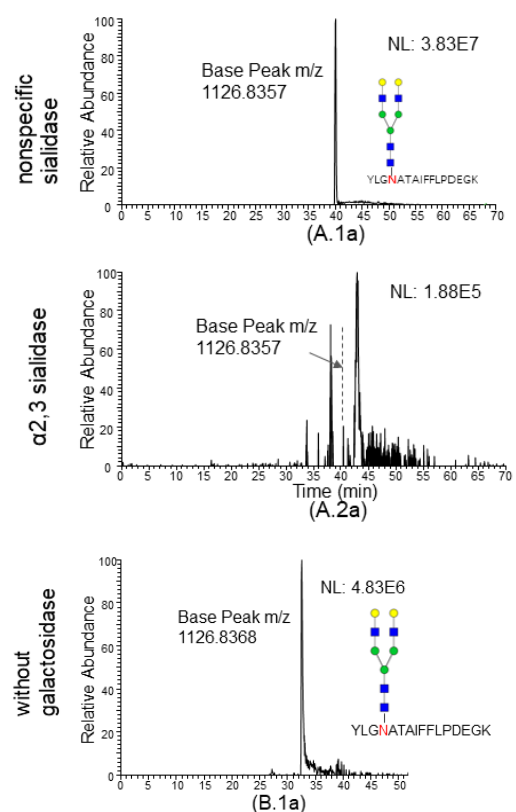

(B)
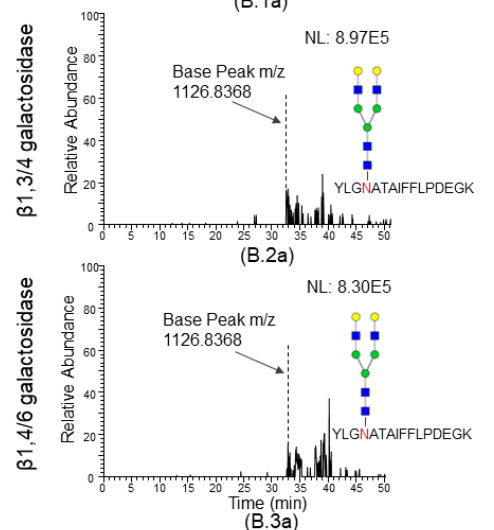
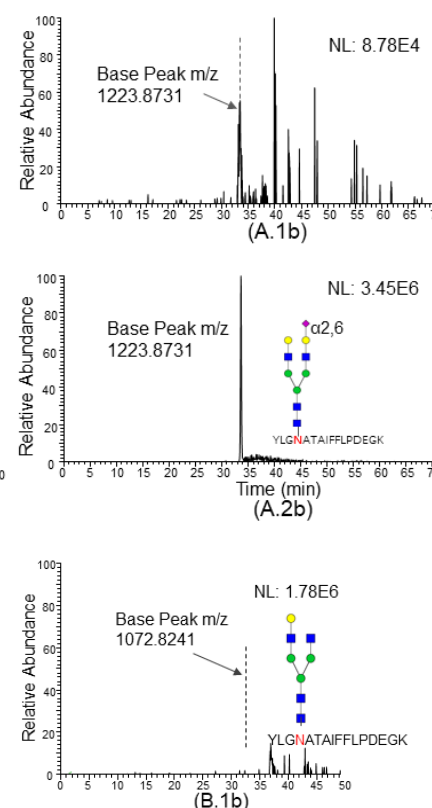

L: 3.45E6
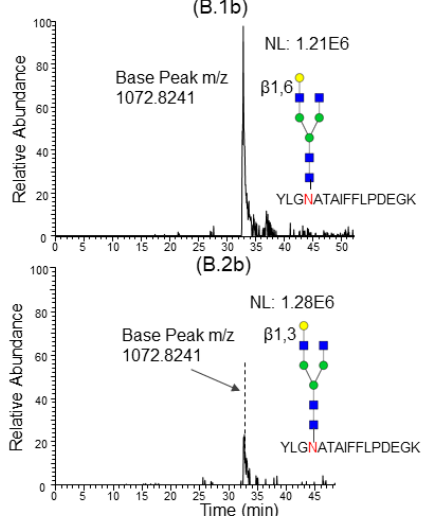
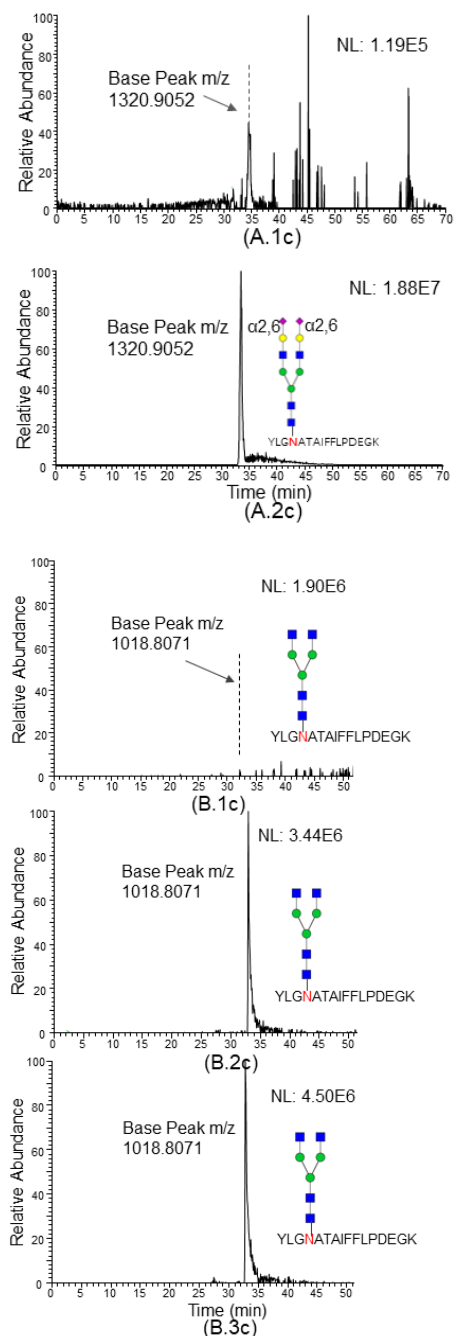

Supporting Figure S9. The extracted chromatogram of glycopeptides with various glycosidase digestions. The commercial A1AT standard from human serum was first digested to glycopeptides with trypsin. (A) It is digested with non-specific sialidase or $\alpha 2,3$ specific sialidase to distinguish the relative abundance of $\alpha 2,3 / 6$ sialic acid linkages. (B) It is then digested with non-specific sialidase, followed by digestion with $\beta 1,3 / 4$ galactosidase and $\beta 1,4 / 6$ galactosidase respectively to distinguish the relative abundance of $\beta 1,3 / 4 / 6$ linkages. 
nLTEIPE_54201/nLTEIPE_54200

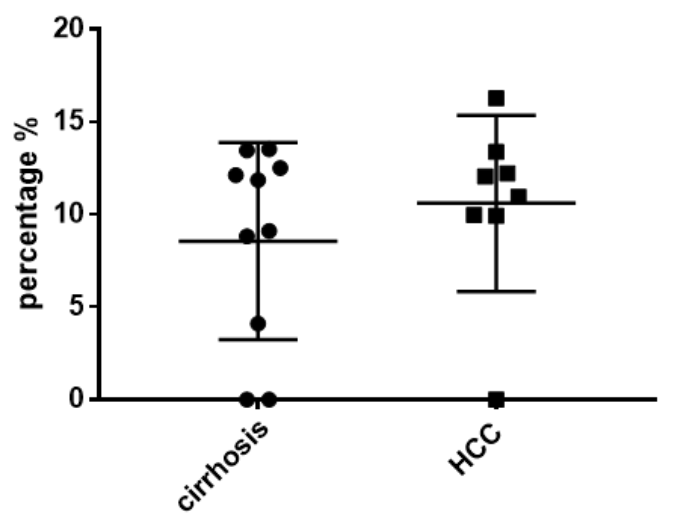

(A)
nLTEIPE_65301/nLTEIPE_65300

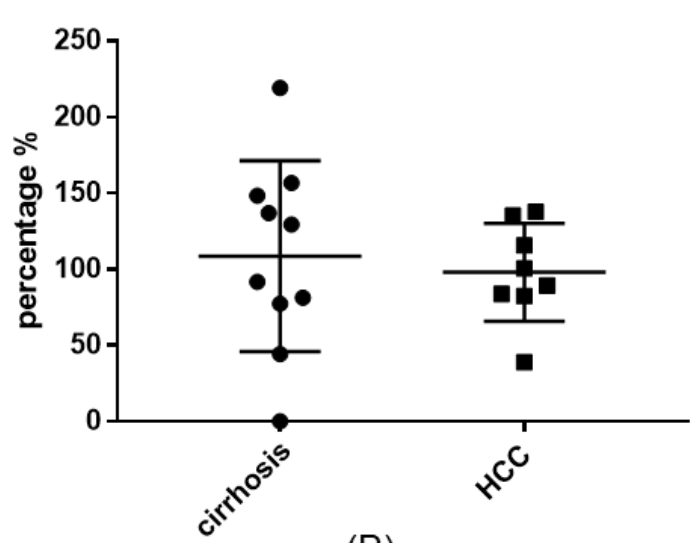

(B)
AHQSnSTNIF_54201/AHQSnSTNIF_54200

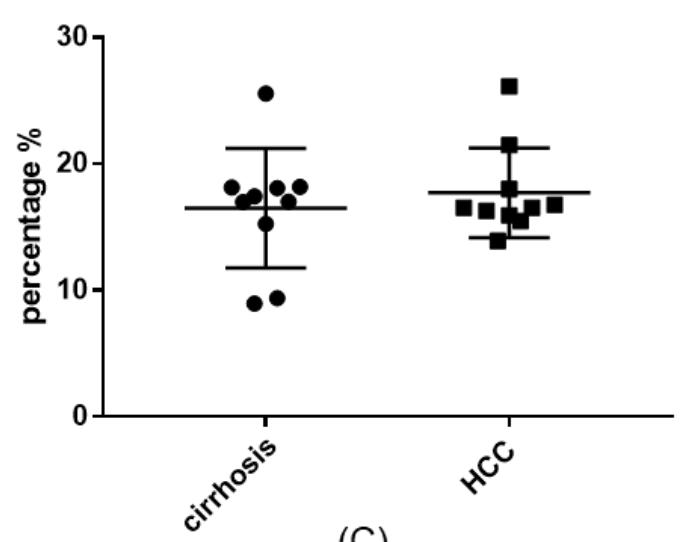

(C)

Supporting Figure S10. Fucosylation level of A1AT at Asn107 (nLTEIPE) and Asn70 (AHQSnSTNIF) did not show differences among early stage HCC and cirrhosis patients. 